\title{
The genetics and screening of familial hypercholesterolaemia
}

\author{
Raymond Henderson ${ }^{1}$, Maurice O'Kane ${ }^{2}$, Victoria McGilligan ${ }^{1}$ and Steven Watterson ${ }^{1 *}$
}

\begin{abstract}
Familial Hypercholesterolaemia is an autosomal, dominant genetic disorder that leads to elevated blood cholesterol and a dramatically increased risk of atherosclerosis. It is perceived as a rare condition. However it affects 1 in 250 of the population globally, making it an important public health concern. In communities with founder effects, higher disease prevalences are observed.

We discuss the genetic basis of familial hypercholesterolaemia, examining the distribution of variants known to be associated with the condition across the exons of the genes $L D L R, A p O B, P C S K 9$ and $L D L R A P 1$. We also discuss screening programmes for familial hypercholesterolaemia and their cost-effectiveness. Diagnosis typically occurs using one of the Dutch Lipid Clinic Network (DCLN), Simon Broome Register (SBR) or Make Early Diagnosis to Prevent Early Death (MEDPED) criteria, each of which requires a different set of patient data. New cases can be identified by screening the family members of an index case that has been identified as a result of referral to a lipid clinic in a process called cascade screening. Alternatively, universal screening may be used whereby a population is systematically screened.

It is currently significantly more cost effective to identify familial hypercholesterolaemia cases through cascade screening than universal screening. However, the cost of sequencing patient DNA has fallen dramatically in recent years and if the rate of progress continues, this may change.
\end{abstract}

Keywords: Familial hypercholesterolaemia, FH, cascade screening, screening, cholesterol, universal screening, atherosclerosis, CVD, CHD

\section{Background}

Familial Hypercholesterolaemia (FH, OMIM \#143890) is a common genetic cause of premature Coronary Heart Disease (CHD). It is an autosomal, dominant, inherited disorder of lipoprotein metabolism that results in a raised Low Density Lipoprotein Cholesterol (LDL-C) plasma concentration.

Heterozygous $\mathrm{FH}(\mathrm{HeFH})$ is the most common monogenic disorder, affecting 1 in 200-250, twice as high as previously thought [1], with a penetrance of greater than $90 \%$ [2]. It is believed that there are 34 million $\mathrm{FH}$ cases worldwide $[1,3]$ and that less than $1 \%$ of potential patients with $\mathrm{FH}$ have been identified in most countries [3].

\footnotetext{
* Correspondence: s.watterson@ulster.ac.uk

'Northern Ireland Centre for Stratified Medicine, Ulster University, C-TRIC, Altnagelvin Hospital Campus, Derry, Co Londonderry, Northern Ireland BT47 6SB, UK

Full list of author information is available at the end of the article
}

If $\mathrm{HeFH}$ is left untreated, there is a significant likelihood of CHD onset prior to age 55 (men) and 60 (women). Half of all untreated $\mathrm{HeFH}$ men and $15 \%$ of women will die of CHD-induced myocardial infarction (MI) before these ages [3, 4]. Homozygous $\mathrm{FH}(\mathrm{HoFH})$ is rare with an estimated global prevalence of 1/160,000-300,000 [5]. However, when left untreated, patients with $\mathrm{HoFH}$ can succumb to MI as teenagers [4] with one reported case of a 4 year old child dying from CHD-induced MI [6].

In certain populations, the frequency of heterozygous FH may be markedly higher than 1 in 200 . When a population is descended from a small number of colonizing ancestors amongst whom the prevalence of the condition was high, a founder effect occurs. Such founder effects are thought to be responsible for the prevalence of $\mathrm{FH}$ associated variants amongst Finns, Icelanders, Christian Lebanese, Tunisians, Gujarati South African Indians, Ashkenazi 
Jews, South African Afrikaners and French Canadians [7] that is as high as 1 in 67 for Ashkenazi Jews. Homozygous $\mathrm{FH}(\mathrm{HoFH})$ has been recorded as ten-fold higher in founder populations, principally due to consanguineous marriages [8].

Hydroxymethylglutaryl coenzyme A (HMGCoA) reductase inhibitors (statins) [9-11] are now the first line treatment for $\mathrm{HeFH}$ and $\mathrm{HoFH}$. Prior to their emergence, mortality rates resulting from $\mathrm{CHD}$ in $\mathrm{FH}$ patients were nearly 100 -fold greater in young adults aged 20-39, and approximately 4-fold greater in patients aged 40-59 than background [12]. However, there exists potential for improvement in the current detection and management of $\mathrm{FH}$. Of those diagnosed, it has been shown that currently only 10-25\% receive appropriate therapy [13].

Here, we review the genetics of $\mathrm{FH}$ and the efficacy of $\mathrm{FH}$ screening programmes. We suggest that the expansion of screening programmes has the potential to contribute significant economic and social benefit.

\section{Review}

\section{The genetic basis of $\mathrm{FH}$}

Elevated cholesterol was first demonstrated as a major risk factor for CHD in 1961 [14]. Lipoproteins were subsequently identified as a factor in atherosclerosis [15] and they were classified into the following cholesterolcarrying types in order of increasing density: chylomicrons, very-low-density lipoprotein (VLDL), Low Density Lipoprotein (LDL), intermediate-density lipoprotein (IDL) and high-density lipoprotein (HDL). The regulation of cholesterol via the LDL-receptor (LDLR) pathway featuring receptor-mediated endocytosis was recognised as critical to atherosclerosis [16] and this facilitated the identification of genetic defects that cause malfunction of the LDL receptor as a major risk factor [17].

The majority of FH cases are caused by mutations in the $L D L R$ gene, resulting in defective synthesis, assembly, transport, recycling or vesicle formation (Fig. 1). Mutations in the $L D L R$ gene cause $\mathrm{FH}$ in $79 \%$ of cases. Apolipoprotein B (ApoB) helps the LDL-receptor bind LDL and mutations in $A p o B$ account for $\sim 5 \%$ of $\mathrm{FH}$ cases.

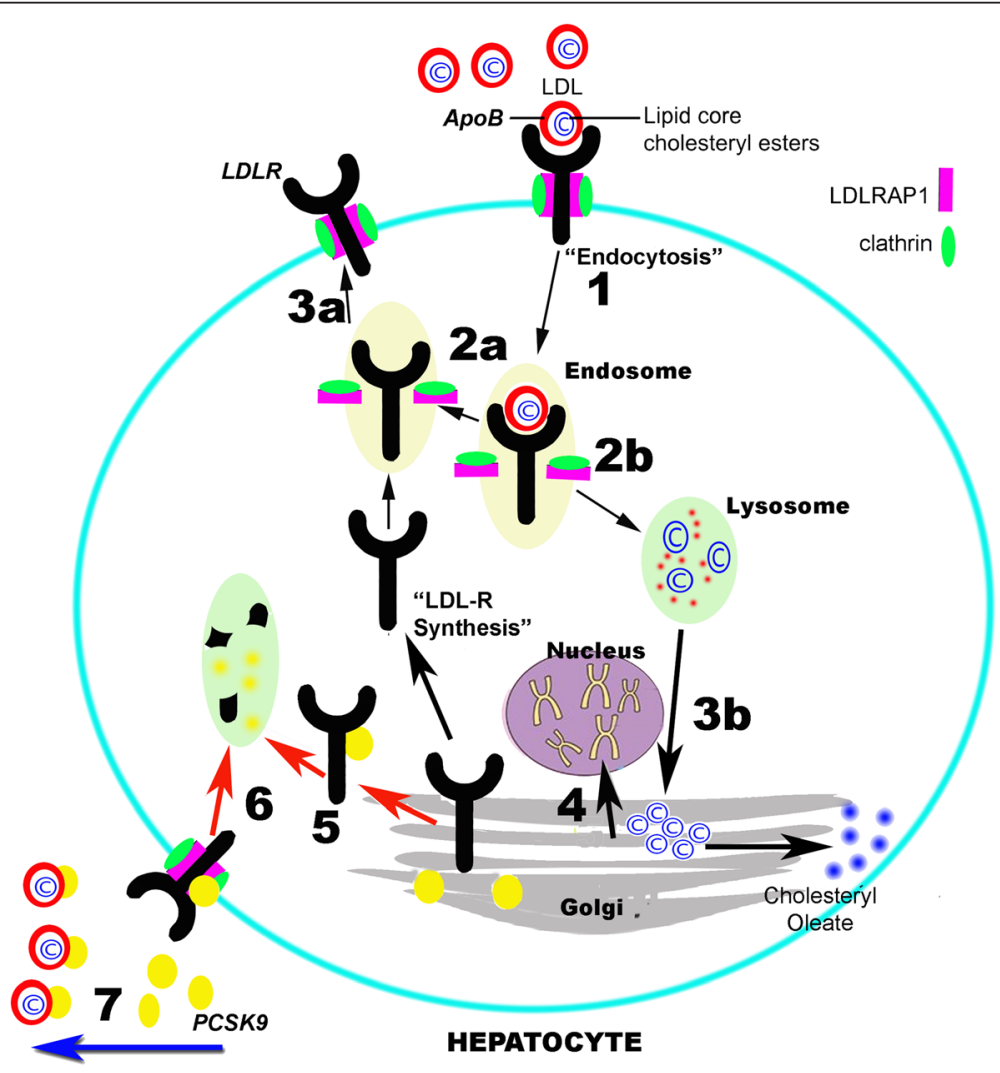

Fig. 1 The LDLR pathway. The LDL receptor (LDLR), part of a LDLR/clathrin/LDLRAP1(ARH) vesicle, binds to the ApoB in LDL particles, internalsing them (1) [26]. The receptor-ligand complex dissociate and LDLR is either recycled (2a and 3a) or degraded (2b and 3b). Residual cholesterol levels regulate the transcription of LDLR (4). PCSK9 is endogenously secreted from the Golgi apparatus where it binds to LDLR (5) [93]. Alternatively, PCSK9 can exogenously bind to LDLR (6). Once internalised to the hepatocyte, PCSK9 directs bound LDLR to the lysosome for degradation. Recent evidence suggests that PCSK9 can bind to LDL via ApoB in free circulation (7) [94] 
Proprotein convertase subtilisin/kexin type 9 (PCSK9) degrades the LDL-receptor and gain of function mutations in PCSK9 account for $<1 \%$ of FH cases [18]. A very rare recessive form of $\mathrm{FH}$ is caused by mutations in low-density lipoprotein receptor adaptor protein 1 (LDLRAP1). The remaining $15 \%$ of $\mathrm{FH}$ cases are either polygenic or are driven by monogenic mutations whose prevalence is not yet determined [18]. The latter include mutations in $A P O E$ [19], APOB [20], SREBP2 [21] and STAP1 [22].

The $\mathrm{FH}$ variant database maintained as part of the Leiden Open Variation Database (LOVD) stores the number of sequence variants for LDLR, PCSK9, and LDLRAP1 [23-25]. Our discussion of the genetic basis of FH describes data from the current release of the LOVD. However, the number of reported variants and our understanding of their role is likely to develop as a result of future studies.

\section{LDLR}

To date research has uncovered a large number of mutations in the LDL-receptor protein associated with $\mathrm{FH}$.
The LOVD FH variant database describes 1741 mutations (retrieved 3rd July 2015). Of these, 1295 are understood to be unique variants, with 1064 predicted to be pathogenic, 143 predicted to be non-pathogenic, and 88 of unknown significance (personal communication, Dr. Sarah Leigh, 13 July 2015). Mutations can yield FH through a range of mechanisms. These include affecting splicing of the pre-messenger RNA (pre-mRNA), altering the promoter region that affects gene transcription, through single amino acid substitutions, creating premature stop codons and introducing large rearrangements. These mutations affect the structure and function of the LDL-receptor and range across the entirety of the $L D L R$ gene (Fig. 2), with nearly all amino acid substitutions identified as having deleterious effects. When the mutation occurs as a large rearrangement, or in cysteinerich repeats either as substitutions or premature stop codons, it produces an entirely non-functional protein [26]. Defects in a splice junction beside an exon may or may not effect splicing.

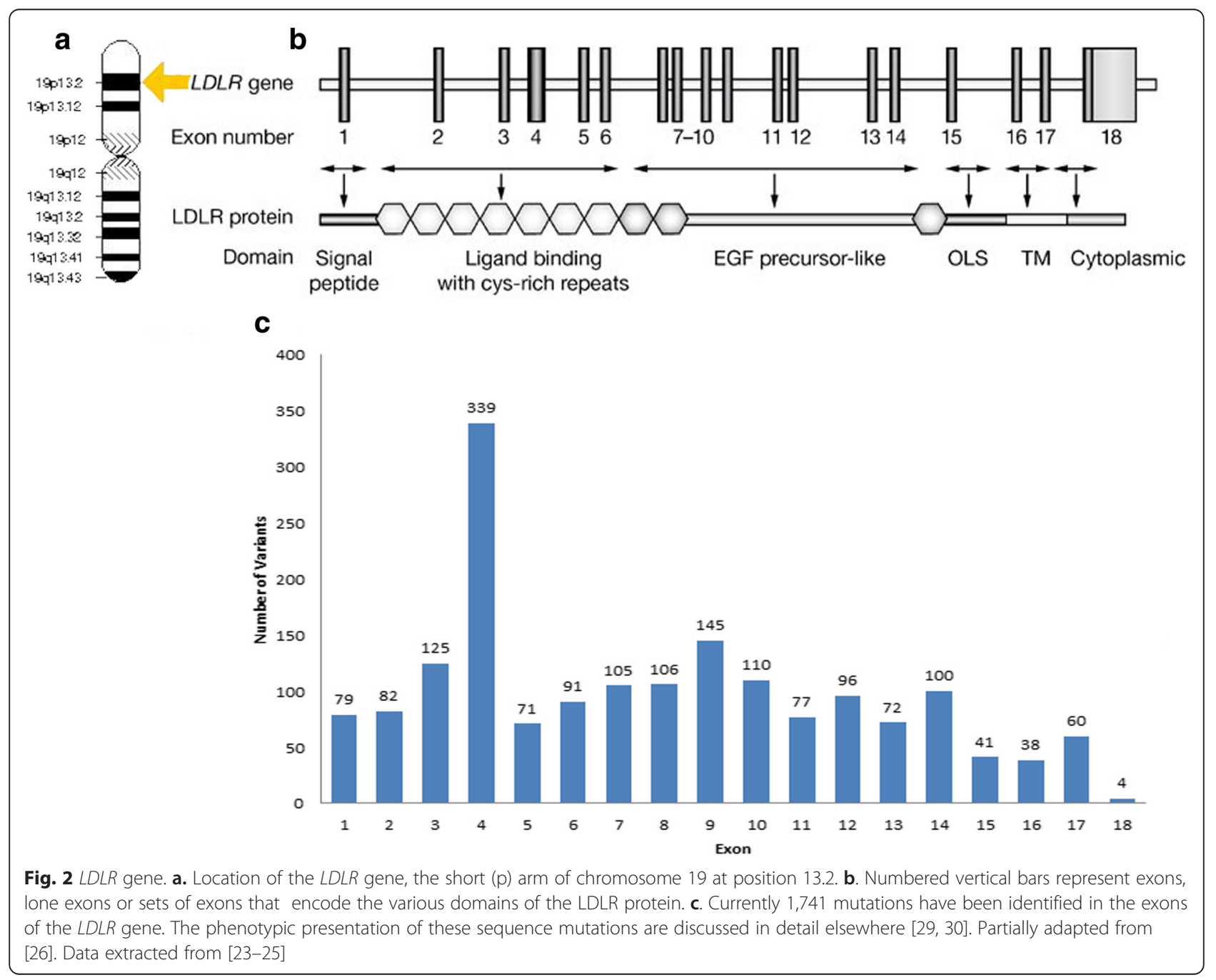




\section{$A p o B$}

$A p o B$ variants are principally located on one exon, number 26 (Fig. 3). Patients with Familial Ligand-Defective Apolipoprotein $\mathrm{B}$ may have a milder form of presentation of FH than that caused by LDLR mutations [27].

\section{PCSK9}

Missense mutations in PCSK9 that cause a gain-of-function lead to a rare form of $\mathrm{FH}$ [28]. Loss-of-function in certain ethnic populations has been shown to result in lower LDL$\mathrm{C}$ levels and protect against CHD [28]. Figure 4 shows both gain-of-function and loss-of-function variants along with variants of unknown significance [23-25, 29, 30].

PCSK9 inhibition/repression has emerged as an important objective in clinical trials where PCSK9 inhibitors have demonstrated significant cholesterol lowering efficacy [31].

\section{LDLRAP1}

LDLRAP1 mutations show a recessive model of inheritance. As such, this rarely-occurring disease is termed autosomal recessive hypercholesterolaemia (ARH, OMIM \#603813) to differentiate it from the
FH conditions attributable to LDLR, PCSK9, and $A$ po $B$ mutations [32]. Figure 5 shows the distribution of known mutations across LDLRAP1. Exon 2 mutations lead to a phenotype similar to HoFH. Mutations in exon 6 have been shown to be more receptive to lipid-lowering therapy [29, 30]. ARH cases generally have lipid levels between those of $\mathrm{HeFH}$ and $\mathrm{HoFH}$ patients and ARH patients tend to be the progeny of ARH consanguineous marriages [33]. HDL levels are greater than those of patients with HoFH. Consequently, early-onset CHD is postponed. Unlike HoFH, no case of a patient under 20 years old with $\mathrm{CHD}$ has been recorded.

\section{Diagnosis}

Identification of $\mathrm{FH}$ is primarily by clinical diagnosis with subsequent confirmation by genetic testing where possible. A family history of premature CHD, a clinical history of premature CHD, physical examination for xanthomas and corneal arcus and elevated plasma LDL$\mathrm{C}$ concentration are all used in diagnosis. These characteristics have been used to develop the most widely used clinical criteria to aid diagnosis: the Dutch Lipid Clinic

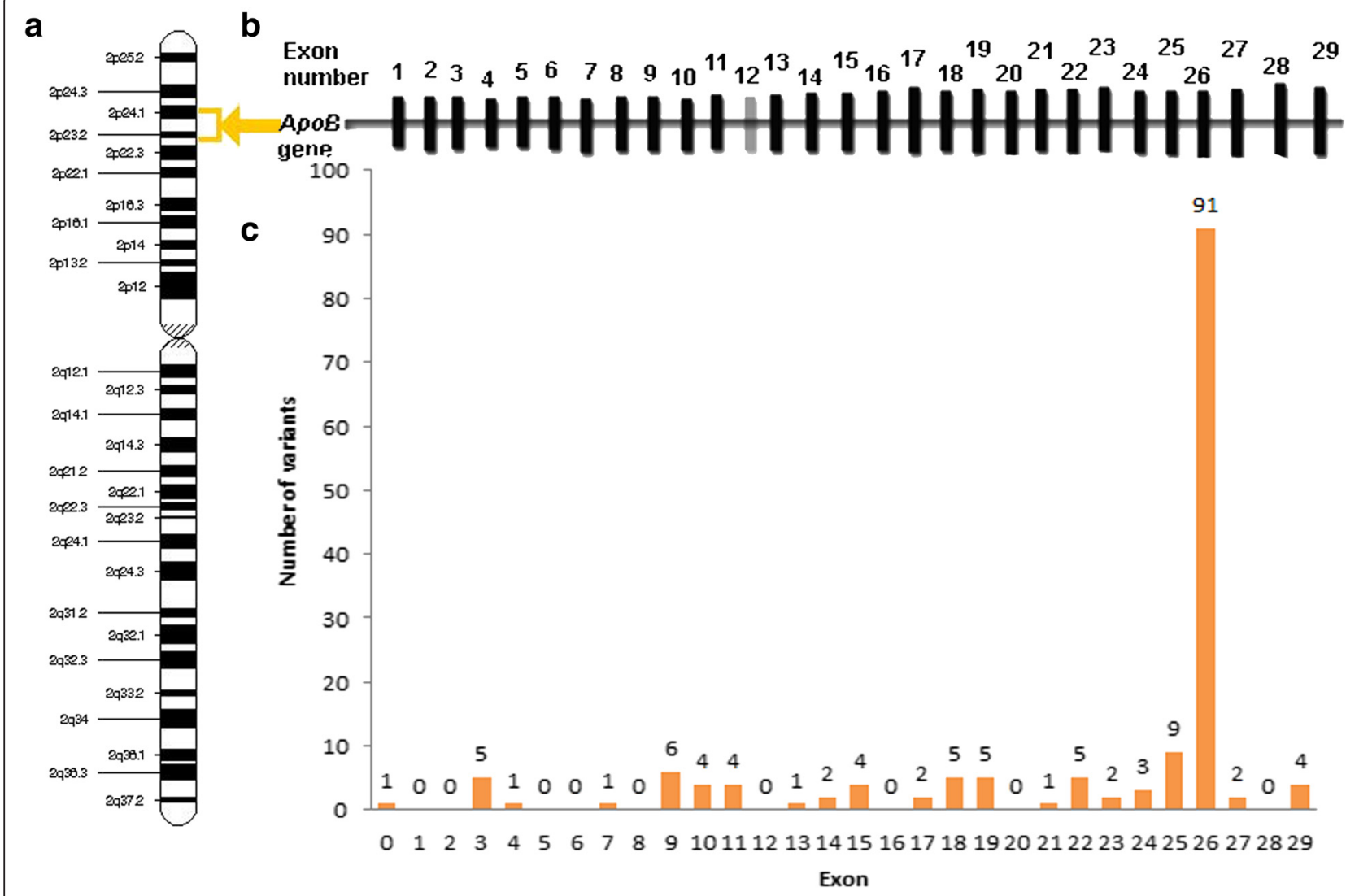

Fig. $3 A p o B$ gene. a. Location of the $A p o B$ gene on the short (p) arm of chromosome 2 between positions 24 and 23. b. Numbered vertical bars representing the exons. c. Only 8 disease-associated sequence variants have been found to occur in $A p o B$, and the majority of these are at the mutational hotspot exon 26. When translated this domain functions as the region for LDLR binding. Data extracted from [23-25] 


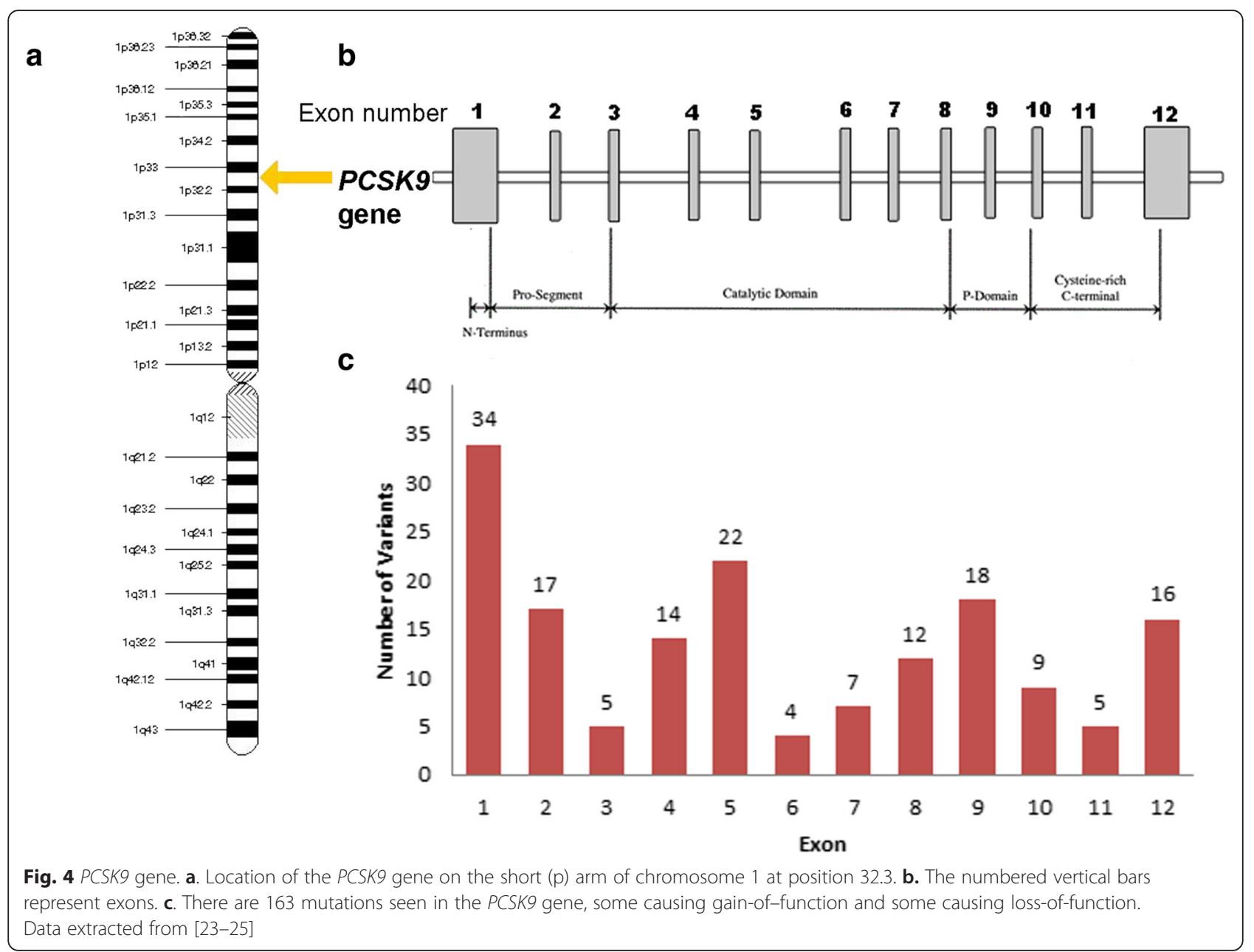

Network (DLCN) criteria, shown in Table 1 [34]. Genetic analysis is suggested if the DLCN score is $>5$. Two other widely used and clinically proven diagnostic tools are the Simon Broome Register (SBR) criteria used in the UK and shown in Table 2 [35] and the Make Early Diagnosis to Prevent Early Death (MEDPED) criteria used in the USA and shown in Table 3 [36]. No international standard currently exists. SBR and DLCN are similar in their choice of criteria, but SBR accepts the presence of a DNA mutation as a definitive confirmation of $\mathrm{FH}$, whereas DLCN needs additional criteria for definite FH diagnosis. For patients with a diagnosis of 'definite' FH by SBR criteria, a causal mutation can be found in greater than $80 \%$ of cases [7, 37]. Each system adopts different cholesterol cut-offs for concluding premature CHD. The MEDPED criteria rely on age-specific and family relative-specific total cholesterol (TC) only. Although easy to use, MEDPED does not incorporate clinical characteristics or identified $\mathrm{FH}$ gene mutations. Their relative merits have been explored in comparative studies [38] and, using a genetic diagnosis as the comparator, SBR criteria have demonstrated a sensitivity of $34 \%$ and a specificity of $89 \%$ for FH diagnosis [39].

Recent research has developed a $\mathrm{FH}$ prognostic model, Familial Hypercholesterolaemia Case Ascertainment Tool (FAMCAT), composed of nine clinical factors, to enhance $\mathrm{FH}$ case identification in primary care [40]. The FAMCAT model may be more sensitive than DLCN, SBR or MEDPED [40] and is based on SBR criteria including family history details such as $\mathrm{MI}, \mathrm{FH}$, and raised cholesterol. However, its clinical utility has yet to be assessed.

There is emerging evidence that a large proportion of patients with a clinical diagnosis of $\mathrm{FH}$ in whom a causative mutation cannot be detected may have polygenic hypercholesterolaemia, i.e. an accumulation of mutations, each of which individually has a small LDL raising effect but which together result in the level of LDL cholesterol elevation typically found in $\mathrm{FH}$ patients [41]. By using a gene loading score based on 12 common LDL-raising Single Nucleotide Polymorphisms (SNPs), it has been shown that mutation negative $\mathrm{FH}$ cases demonstrate a significantly higher LDL gene loading score than in control subjects [41]. 


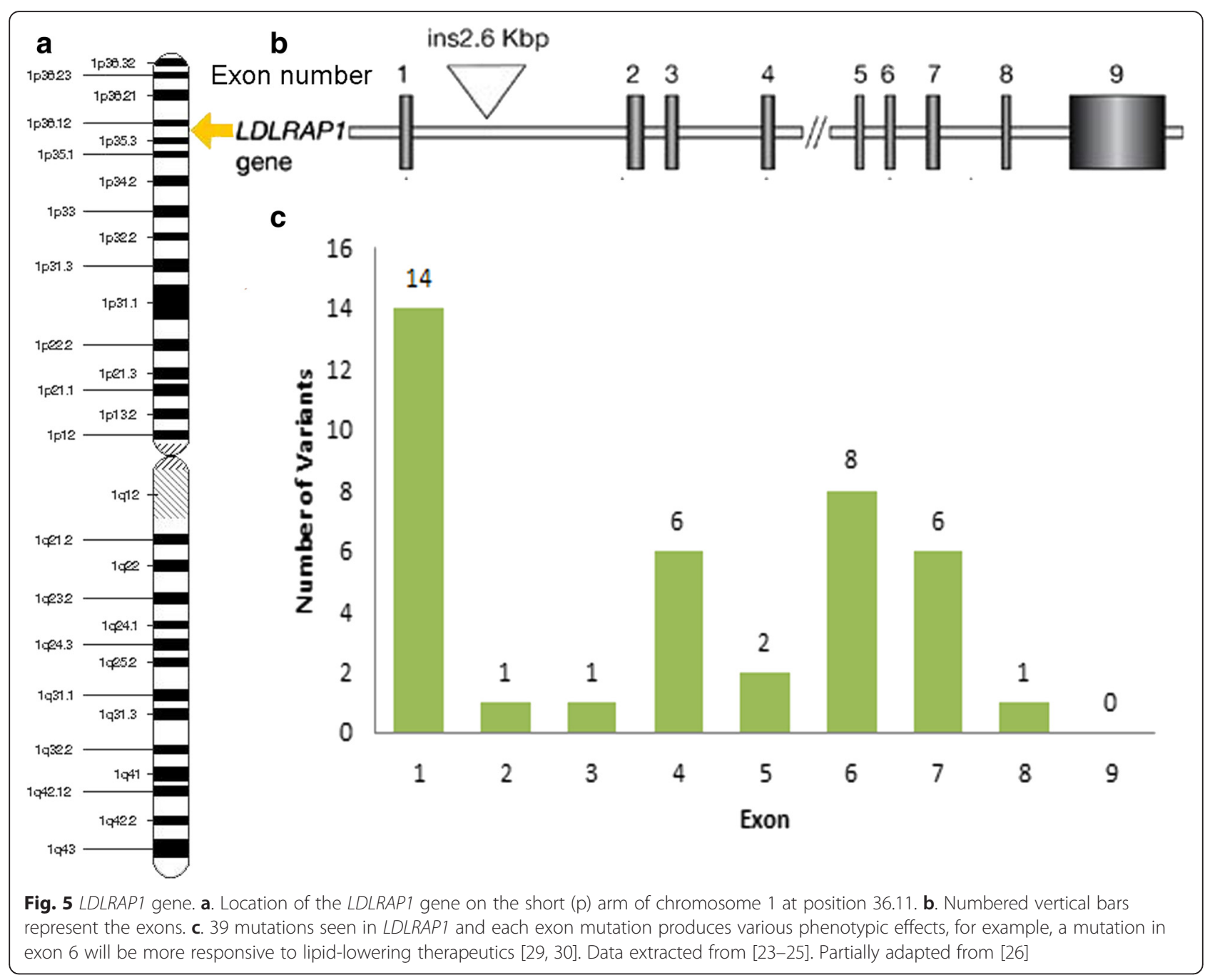

FH must also be differentiated from other dyslipidaemias such as Familial Combined Hyperlipidaemia (FCHL) and polygenic hypercholesterolaemia with increased Lp(a), both of which may be associated with increased vascular risk and may present with a clinical phenotype suggestive of FH. FCHL is a condition in which the patient has serum cholesterol and/or triglyceride concentration exceeding the 90th percentile of the age and sex matched healthy population and raised serum cholesterol and/or triglyceride in at least one first degree relative [42]. FCHL is considered to be the most common inherited lipid disorder and an important risk factor for vascular disease with a prevalence of 10-20\% amongst survivors of myocardial infarction. Although FCHL shows autosomal dominant inheritance with low penetration, the causative genes remain unclear. Associated with polygenic hypercholesterolaemia, Lp(a) is a circulating lipoprotein consisting of an LDL particle covalently bound to apolipoprotein(a). Circulating serum $\mathrm{Lp}(\mathrm{a})$ concentration is determined largely by variation in the apolipoprotein(a) gene and elevated $\mathrm{Lp}(\mathrm{a})$ is an independent risk factor for vascular disease [43]. Patients with polygenic hypercholesterolaemia and increased Lp(a) may therefore be clinically misdiagnosed as FH cases.

In order to diagnose $\mathrm{FH}$, secondary causes of hyperlipidaemia must be ruled out by excluding cholestatic liver disease, hypothyroidism, significant proteinuria, diabetes mellitus and excess alcohol [3].

\section{Treatment}

The National Institute for Health and Care Excellence (NICE) in the UK recommends FH cases should target a reduction in LDL-C levels of over $50 \%$ from baseline (i.e. LDL-C levels before therapy) [44], whereas the European Society of Cardiology/European Atherosclerosis Society (EAS) recommends that the target level for acceptable LDL-C is $<1.8 \mathrm{mmol} / \mathrm{l}$ in $\mathrm{HeFH}$ patients with confirmed $\mathrm{CHD}$, and $<2.5 \mathrm{mmol} / \mathrm{l}$ in $\mathrm{HeFH}$ patients without confirmed CHD [3]. 
Table 1 DLCN Diagnostic Criteria for FH

\begin{tabular}{ll}
\hline Group 1: Family History & Points \\
i. First-degree relative with premature CHD & 1 \\
ii. First-degree relative with $L D L-C>95$ th percentile \\
by age, gender for country \\
iii. First-degree relative with tendinous xanthomata \\
and/or arcus cornealis \\
$\begin{array}{l}\text { iv. Children under } 18 \text { years with } L D L-C>95^{\text {th }} \\
\text { percentile by age, gender for country }\end{array}$ \\
Group 2: Clinical History \\
$\begin{array}{l}\text { i. Premature CHD } \\
\text { ii. Premature cerebrovascular or peripheral vascular disease }\end{array}$ \\
\end{tabular}

Group 3: Physical Examination Points

i. Tendinous xanthomata

ii. Arcus cornealis prior to 45 years

Group 4: LDL-C Levels

i. LDL-C > $8.5 \mathrm{mmol} / \mathrm{l}(\sim 330 \mathrm{mg} / \mathrm{dl})$

ii. LDL-C 6.5-8.4 mmol/l ( 250-329 mg/dl)

iii. LDL-C 5.0-6.4 mmol// ( 190-249 mg/dl)

iv. LDL-C 4.0-4.9 mmol// ( 155-189 mg/dl)

Group 5: DNA Analysis Points

i. Causative mutation in the LDLR, ApoB or PCSK9 gene

Total Score: -

Definite $\mathrm{FH}>8$ points

Probable FH: 6-8 points

Possible FH: $3-5$ points

Unlikely FH: $0-2$ points

Genetic Testing For:-

i. Patients with a score $>5$ points

ii. Patients with an obvious diagnosis of xanthomata with high cholesterol and a CHD family history

Causative Mutation Found:-

Genetic testing for all first degree relatives

${ }^{a} \mathrm{CHD}$ Before age 55 (men), 60 (women)
Cohort comparisons [45] have demonstrated that $\mathrm{HeFH}$ patients treated with either simvastatin or atorvastatin had a $76 \%$ overall risk reduction in CHD and no increased risk of adverse effects associated with statin therapy, including increased plasma liver enzyme activity, myalgia and, less commonly, rhabdomyolysis and myopathy [4]. However, high-intensity statin therapy may be associated with an increased risk of developing type 2 diabetes mellitus [46].

Almost $80 \%$ of $\mathrm{FH}$ patients prescribed statin therapy do not attain the EAS recommended LDL-C levels [47]. In a small number of $\mathrm{FH}$ cases it has been suggested that this is due to statin resistance connected to polymorphisms in a number of genes, although this picture is currently unclear [48]. Alternative hypotheses include non-compliance due to adverse side-effects or patient choice [49].

Ezetimbe, a cholesterol lowering drug that blocks cholesterol absorption in the small intestine may be used in combination with statin therapy in patients who are not achieving lipid targets on statin monotherapy or in patients who are intolerant of statins. Other therapeutic agents include bile acid sequestrants, mipomersen (an inhibitor of apolipoprotein B-100 synthesis), lomitapide (a microsomal triglyceride transfer protein inhibitor), PCSK9 inhibitors [4], Fibrates [50] and Niacin [51], some of which have been demonstrated in combination with statins $[52,53]$. In the case of $\mathrm{HoFH}$, high-intensity statin treatment and LDL apheresis can be used [4].

It has been estimated that $96-98 \%$ of CHD deaths in FH patients aged less than 40 years could potentially be averted with just statin therapy [54].

For children diagnosed with $\mathrm{FH}$, lifestyle and diet are targeted. Many statins are approved for use from the ages of 8-10, although Atorvastatin has been approved for use from age 6 [18]. In the UK, NICE recommends consideration of statin therapy from age 10 years. Ezetimbe has been approved for use from the age of 10

Table 2 Simon Broome Register Diagnostic Criteria

\begin{tabular}{|c|c|}
\hline \multicolumn{2}{|c|}{ A diagnosis of explicit FH requires either (1), (2) or (3) } \\
\hline \multirow[t]{2}{*}{1} & i. Cholesterol higher than $7.5 \mathrm{mmol} / \mathrm{L}$ or $\mathrm{LDL}$-cholesterol above $4.9 \mathrm{mmol} / \mathrm{L}$ in adult \\
\hline & ii. Tendon xanthomas in patient or a 1st degree relative (parent, sibling, child), or in a 2nd degree relative (grand parent, uncle, aunt) \\
\hline \multirow[t]{2}{*}{2} & i. Cholesterol higher than $6.7 \mathrm{mmol} / \mathrm{L}$ or LDL-cholesterol above $4.0 \mathrm{mmol} / \mathrm{L}$ in a child under 16 years of age \\
\hline & ii. Tendon xanthomas in patient or a 1st degree relative (parent, sibling, child), or in a 2nd degree relative (grand parent, uncle, aunt) \\
\hline 3 & i. DNA based evidence of a functional LDLR, PCSK9 and APOB mutation \\
\hline \multicolumn{2}{|c|}{ A diagnosis of probable $\mathrm{FH}$ requires either (1), (2) or (3) } \\
\hline \multirow[t]{2}{*}{1} & i. Cholesterol higher than $7.5 \mathrm{mmol} / \mathrm{L}$ or LDL-cholesterol above $4.9 \mathrm{mmol} / \mathrm{L}$ in adult \\
\hline & ii. Family History of myocardial infarction (MI) before 50 years of age in a 2nd degree relative or below age 60 in a 1st degree relative \\
\hline \multirow[t]{2}{*}{2} & i. Cholesterol higher than $6.7 \mathrm{mmol} / \mathrm{L}$ or LDL-cholesterol above $4.0 \mathrm{mmol} / \mathrm{L}$ in a child under 16 years of age \\
\hline & ii. Family History of myocardial infarction (MI) before 50 years of age in a 2nd degree relative or below age 60 in a 1 st degree relative \\
\hline 3 & $\begin{array}{l}\text { i. A family history of raised total cholesterol - higher than } 7.5 \mathrm{mmol} / \mathrm{L} \text { in adult } 1 \mathrm{st} \text { or } 2 \mathrm{nd} \text { degree relative or higher than } 6.7 \mathrm{mmol} / \mathrm{L} \text { in a child } \\
\text { or sibling aged under } 16 \text { years }\end{array}$ \\
\hline
\end{tabular}


Table 3 The US (MEDPED) Diagnostic Criteria for FH. FH is diagnosed if total cholesterol (TC) levels exceed the threshold stated [95]

\begin{tabular}{llcll}
\hline $\begin{array}{l}\text { Age } \\
\text { (years) }\end{array}$ & $\begin{array}{l}\text { First Degree } \\
\text { relative with } \\
\text { FH (TC, mmol/L) }\end{array}$ & $\begin{array}{l}\text { Second Degree } \\
\text { relative with FH } \\
(\mathrm{TC}, \mathrm{mmol} / \mathrm{L})\end{array}$ & $\begin{array}{l}\text { Third Degree } \\
\text { relative with } \\
\mathrm{FH}(\mathrm{TC}, \mathrm{mmol} / \mathrm{L})\end{array}$ & $\begin{array}{l}\text { General } \\
\text { Population } \\
(\mathrm{TC}, \mathrm{mmol} / \mathrm{L})\end{array}$ \\
\hline$<20$ & 5.7 & 5.9 & 6.2 & 7 \\
$20-29$ & 6.2 & 6.5 & 6.7 & 7.5 \\
$30-39$ & 7 & 7.2 & 7.5 & 8.8 \\
$\geq 40$ & 7.5 & 7.8 & 8 & 9.3 \\
\hline
\end{tabular}

in the USA and Europe and bile acid sequestrants have been approved for use from age 10 in the USA. Screening is recommended from the age of 5 although this can be complicated by issues around parental consent [18].

\section{Screening}

FH has no formal disease classification under current WHO disease classifications [3, 55]. Cascade screening (CS), whereby family members are traced from an established $\mathrm{FH}$ index case, is more cost effective than any other screening strategy currently available [56] and is recommended in NICE guidelines [44]. Approximately half of the first degree relatives of an index case will be found to have the FH mutation [57]. NICE recommends against using SBR criteria for case detection of relatives of an index case as this results in under-diagnosis [44] and instead to use genetic testing (where the causative mutation in the index case has been identified) or to use age- and gender-specific LDL-C concentration where a genotypic result is not available in the index case [58].

At the introduction of CS in the Netherlands, 2039 relatives of 237 index cases were found to have $\mathrm{FH}$ with $39 \%$ already taking treatment. A year later this had risen to $93 \%$ [59]. To date, approximately 23,000 FH cases have been determined in the Netherlands by CS alone [17]. CS has also proven to be effective in Australia and Brazil, where each index case typically yields a further 2 cases $[60,61]$. However, in the UK, it has proven less effective, yielding between 0.4 and 0.7 new cases per $\mathrm{FH}$ index case [57, 62, 63]. CS has still demonstrated its clinical utility in the UK [64], decreasing the age of $\mathrm{FH}$ diagnosis and increasing the number of people with $\mathrm{FH}$ on statin therapy [45].

It has been shown that even with systematic CS through to 3rd degree relatives, in a 'best case' detection scenario of 8.6 relatives per index case as in the Netherlands model [59], 17 \% of FH cases must be identified as index cases in order to achieve a detection rate of $80 \%$ of putative FH cases after introducing CS [65].

An alternative to CS is universal screening (UScr) in which a population is systematically screened. This has not yet been applied to $\mathrm{FH}$, but could be undertaken by cholesterol measurement or genotyping in childhood. Genotyping might be more effective for populations in which founder effects occur with a restricted number of prevalent mutations. UScr has been a great public health triumph for detecting and treating disorders such as phenylketonuria (PKU), medium-chain acyl-CoA dehydrogenase deficiency (MCAD), cystic fibrosis (CF) [66] and cervical cancer [67]. The newborn screening programmes implemented for $\mathrm{CF}$ have shown direct benefits such as preventing malnutrition [68] and may lead to indirect benefits such as informing parental reproductive choices, reducing parental stress and facilitating clinical trial recruitment [69]. Such advantages have led some to advocate genome wide analyses from a single sample although there are significant ethical and regulatory considerations to doing so [70].

International guidelines advocate targeted screening for the identification of new $\mathrm{FH}$ index cases [71] in which screening can be directed at specific patient groups likely to show a high prevalence of $\mathrm{FH}$ such as those post acute coronary syndrome. A study based around an Australian coronary care unit demonstrated that the prevalence of possible/definite $\mathrm{FH}$ (as defined by DLCN criteria) was as high as $14.3 \%$ in patients below the age of 60 with a current or prior history of coronary artery disease [72]. Such targeted screening is an effective strategy for identifying new FH index cases.

It has been suggested that new FH index cases could be identified systematically from electronic health records [73, 74], but a preliminary study yielded disappointing results with only 2 new definite FH index cases identified from a population of 12,100 [75]. However, a strategy that would likely yield a higher FH detection rate is Reverse Cascade Screening (RCS) [76]. This combines elements of UScr and CS and involves screening an infant's total cholesterol (TC) when they receive vaccinations at 15 months of age. Following the identification of elevated cholesterol (here defined as 1.5 times the median for age), DNA analysis can be utilised to identify relevant mutations and, should they exist, the parents and grandparents would be tested subsequently. It has been suggested that by running this programme for one generation, most, if not all, FH cases would be detected and registered [65].

Screening has conventionally been undertaken by array or PCR amplification. Sanger sequencing has demonstrated its value, but has proven prohibitively expensive. However, Next Generation Sequencing (NGS) with its ability to undertake parallel sequencing relatively quickly, has shown great promise [77]. NGS has demonstrated high levels of specificity and sensitivity [78] in particular when combined with clinical criteria $[79,80]$. 
The value of screening for $\mathrm{FH}$ mutations is not without controversy. As $\mathrm{FH}$ is only an indicator for likely elevated LDL-C and a proportion of elevated LDL-C cases are negative for the canonical variants, it has been argued that screening should focus on phenotype rather than genotype, both for the identification of index cases and in cascade screening, and that a focus on genetic screening can offer false reassurances to variant-negative patients who might still be at risk [81, 82]. The guidelines for screening of $\mathrm{FH}$, provided by the National Lipid Association (USA), still focus principally on phenotypic diagnosis [83].

\section{Economics}

Detection and treatment of $\mathrm{FH}$ leads to significant savings in healthcare costs [56]. In the UK, it is estimated that the identification and optimal treatment of all FH cases would save the NHS $£ 380$ million over a 55 year period, or $£ 6.9$ million/year [84]. When extrapolated to the EU, the savings would yield about $€ 86$ million per year [18]. NICE guidelines estimate that CS leads to an incremental cost effectiveness ratio (ICER) of $£ 2,700$ per quality adjusted life year (QALY). This intervention is considerably less expensive than the $£ 20,000$ to $£ 30,000$ per QALY ceiling that NICE defines as costeffective [85]. Furthermore, the cost of FH testing is likely to drop by as much as four-fold with the introduction of next-generation sequencing [86] improving the cost effectiveness further. It has been estimated that CS and high-intensity statin therapy would lead to 101 fewer deaths/1000 FH patients by CHD [84].

\section{Discussion}

UScr as a general population disease identification strategy has pitfalls in terms of cost and the role of unknown causative variants. However as sequencing costs continue to drop, it is likely that new mechanisms of action will be uncovered and doctors can protect the patient from the irresolution in the genomic data [87].

Several authors propose that the ideal screening scenario is the integration of CS and UScr strategies $[2,88]$. Others posit that as the costs of disease management increases, while the cost of diagnostics decreases, UScr will become more cost-effective and attractive, rendering CS less attractive [89]. The hybrid proposal of RCS [76] may be more cost-effective than UScr and become more so if DNA sequencing continues to outpace Moore's Law in terms of better, cheaper, faster performance [90, 91].

Early recognition of a child with $\mathrm{FH}$, coupled with therapy from a young age, will impede, if not arrest, the onset of atherosclerosis [18]. UScr is already used for conditions such as phenylketonuria, which has a prevalence of 1 per 10,000 , so the societal barriers to UScr should be lower for diseases such as $\mathrm{FH}$ where it has been demonstrated that testing and treating is clearly beneficial [89].

In the Netherlands, children with FH who have received counselling as part of their early intervention therapy have not taken up smoking, a lifestyle risk factor, in $100 \%$ of cases. Additionally, these children have been shown to cope effectively with their diagnosis $[18,92]$.

\section{Conclusion}

Familial Hypercholesterolaemia is a significant risk factor for cardiovascular disease, the leading cause of death globally. Familial Hypercholesterolaemia is an autosomal, dominant genetic disorder predominantly associated with pathogenic variants in the genes $L D L R, A p o B, L D L R A P 1$ and gain of function variants in PCSK9. Screening typically occurs using the Dutch Lipid Clinic Network, the Simon Broome Register or the Make Early Diagnosis to Prevent Early Death (MEDPED) criteria. Typically, a diagnosed case forms an index case from which a cascade screen is undertaken within the same family to identify as many new cases as possible. However, alternative screening programmes include systematic screens across a population (universal screening) and hybrid schemes in which cascade screening is applied to systematically screened subpopulations (reverse cascade screening). Cost-effectiveness is dependent on the scheme. Cascade screening in its typical form is highly cost-effective, although more systematic programmes may become more competitive if genome sequencing costs continue to fall.

The ability to identify $\mathrm{FH}$ patients at the earliest opportunity is both economically and socially beneficial with implications for mortality and morbidity.

Whichever screening strategy is optimal, studies of screening programmes will not only address $\mathrm{FH}$ as one of the world's most prevalent and treatable inherited diseases, but have the potential to contribute to broader studies of hereditary diseases with similar traits.

\section{Abbreviations}

ApoB: Apolipoprotein B; ARH: Autosomal Recessive Hypercholesterolaemia; CHD: Coronary Heart Disease; CS: Cascade Screening; DLCN: Dutch Lipid Clinic Network; EAS: European Atherosclerosis Society; ESC: European Society of Cardiology; FAMCAT: Familial Hypercholesterolaemia Case Ascertainment Tool; FCHL: Familial Combined Hyperlipidaemia; FH: Familial

Hypercholesterolaemia; HDL: High Density Lipoprotein; HeFH: Heterozygous FH; HMGCoA: Hydroxymethylglutaryl coenzyme A; HoFH: Homozygous FH; ICER: Incremental Cost Effectiveness Ratio; IDL: Intermediate Density Lipoprotein; LDL: Low Density Lipoprotein; LDL-C: Low Density Lipoprotein Cholesterol; LDLR: Low Density Lipoprotein Receptor; LDLRAP1: Low Density Lipoprotein Receptor Adaptor Protein 1; LGY: Life Year Gained; LOVD: Leiden Open Variation Database; LP(a): Lipoprotein(a); MEDPED: Make Early

Diagnosis to Prevent Early Death; MI: Myocardial Infarction; NGS: Next Generation Sequencing; NHS: National Health Service (UK); NICE: National Institute for Clinical Excellence; PCSK9: Protein Convertase Subtilison/ Kexin Type 9; QALY: Quality Adjusted Life Year; RCS: Reverse Cascade Screening; SNP: Single Nucleotide Polymophism; TC: Total Cholesterol; UScr: Universal Screening; VLDL: Very Low Density Lipoprotein; WHO: World Health Organisation. 


\section{Competing interests}

The authors declare that they have no competing interests.

\section{Authors' contributions}

$\mathrm{RH}$ undertook literature review, data interpretation, writing and figure design. MOK undertook writing, data interpretation. VM undertook writing and data interpretation, SW undertook writing, data interpretation and integration. All authors read and approved the final manuscript.

\section{Acknowledgements}

We would like to thank Wendy Golding Henderson for her assistance in drafting the manuscript and Sarah Leigh for her assistance with the LOVD FH database and for providing valuable feedback during the drafting of the manuscript.

This work was part financed by a grant awarded to AJ Bjourson under the European Union Regional Development Fund (ERDF) EU Sustainable Competitiveness Programme for N. Ireland \& the Northern Ireland Public Health Agency (HSC R\&D).

\section{Author details}

'Northern Ireland Centre for Stratified Medicine, Ulster University, C-TRIC, Altnagelvin Hospital Campus, Derry, Co Londonderry, Northern Ireland BT47 6SB, UK. ${ }^{2}$ Department of Clinical Chemistry, Altnagelvin Hospital, Western Health and Social Care Trust, Londonderry, Northern Ireland BT47 6SB, UK.

\section{Received: 16 December 2015 Accepted: 3 April 2016}

\section{Published online: 16 April 2016}

\section{References}

1. Sjouke B, Kusters DM, et al. Homozygous autosomal dominant hypercholesterolaemia in the Netherlands: prevalence, genotype-phenotype relationship, and clinical outcome. Eur Heart J. 2015;36(9):560

2. Ademi Z, Watts GF, et al. Cascade screening based on genetic testing is cost-effective: evidence for the implementation of models of care for familial hypercholesterolemia. J Clin Lipidol. 2014:8(4):390-400.

3. Nordestgaard BG, Chapman MJ, et al. Familial hypercholesterolaemia is underdiagnosed and undertreated in the general population: guidance for clinicians to prevent coronary heart disease. Eur Heart J. 2013;34(45):3478-90.

4. Reiner Z. Management of patients with familial hypercholesterolaemia. Nat Rev Cardiol. 2015:12:565-75.

5. Cuchel M, Bruckert E, et al. Homozygous familial hypercholesterolaemia: new insights and guidance for clinicians to improve detection and clinical management. A position paper from the Consensus Panel on Familial Hypercholesterolaemia of the European Atherosclerosis Society. Eur Heart J. 2014;35(32):2146-57.

6. Widhalm K, Binder CB, et al. Sudden death in a 4-year-old boy: a near-complete occlusion of the coronary artery caused by an aggressive low-density lipoprotein receptor mutation (W556R) in homozygous familial hypercholesterolemia. J Pediatr. 2011;158(1):167.

7. Austin MA, Hutter CM, et al. Genetic causes of monogenic heterozygous familial hypercholesterolemia: a HuGE prevalence review. Am J Epidemiol. 2004;160(5):407-20.

8. Izar MC, Machado VA, Fonseca FA. Genetic screening for homozygous and heterozygous familial hypercholesterolemia. Appl Clin Genet. 2010;3:147.

9. Watterson S, Guerriero ML, et al. A model of flux regulation in the cholesterol biosynthesis pathway: Immune mediated graduated flux reduction versus statin-like led stepped flux reduction. Biochimie. 2013;95(3):613-21.

10. Mazein A, Watterson S, et al. A comprehensive machine-readable view of the mammalian cholesterol biosynthesis pathway. Biochem Pharmacol. 2013;86(1):56-66

11. Lu H, Talbot S, et al. Rapid proteasomal elimination of 3-hydroxy-3methylglutaryl-CoA reductase by interferon- $\gamma$ in primary macrophages requires endogenous 25-hydroxycholesterol synthesis. Steroids. 2015;99B: 219-29

12. Neil A, Cooper J, et al. Reductions in all-cause, cancer, and coronary mortality in statin-treated patients with heterozygous familial hypercholesterolaemia: a prospective registry study. Eur Heart J. $2008 ; 29(21): 2625-33$.
13. Hardcastle SJ, Legge E, et al. Patients' perceptions and experiences of familial hypercholesterolemia, cascade genetic screening and treatment. Int J Behav Med. 2014;22(1):92-100.

14. Kannel WB, Dawber TR, et al. Factors of risk in the development of coronary heart disease-six-year follow-up experience: the Framingham Study. Ann Intern Med. 1961;55(1):33-50.

15. Goldstein $J$, Brown MS. Lipoprotein receptors, cholesterol metabolism, and atherosclerosis. Arch Pathol. 1975;99(4):181-4.

16. Brown MS, Goldstein JL. Receptor-mediated control of cholesterol metabolism. Science. 1976;191(4223):150-4.

17. Brown MS, Goldstein JL. A receptor-mediated pathway for cholesterol homeostasis. Science. 1986;232(4746):34-47.

18. Wiegman A, Gidding SS, et al. Familial hypercholesterolaemia in children and adolescents: gaining decades of life by optimizing detection and treatment. Eur Heart J. 2015:36(36):2425-37.

19. Cenarro A, Etxebarria A, et al. The p.Leu167del mutation in APOE gene causes autosomal dominant hypercholesterolemia by down-regulation of LDL receptor expression in hepatocytes, J Clin Endocrinol Metab. 2016; jc20153874 (in press).

20. Alves A, Etxebarria A, et al. Novel functional APOB mutations outside LDL-binding region causing familial hypercholesterolaemia. Hum Mol Genet. 2014;23(7):1817-28.

21. Miserez $R$, Muller $P$, et al. Sterol-regulatory element-binding protein (SREBP)-2 contributes to polygenic hypercholesterolaemia. Atherosclerosis. 2002;164(1):15-26.

22. Fouchier S, Dallinga-Thie $\mathrm{G}$, et al. Mutations in STAP1 are associated with autosomal dominant hypercholesterolemia. Circ Res. 2014;115(6):552-5.

23. Leigh SEA, Leren TP, Humphries SE. Commentary PCSK9 variants: A new database. Atherosclerosis. 2009;203(1):32-3.

24. Leigh SEA, Foster $\mathrm{AH}$, et al. Update and analysis of the University College London low density lipoprotein receptor familial hypercholesterolemia database. Ann Hum Genet. 2008;72(4):485-98.

25. Usifo E, Leigh SEA, et al. Low-density lipoprotein receptor gene familial hypercholesterolemia variant database: update and pathological assessment. Ann Hum Genet. 2012;76(5):387-401.

26. Soutar AK, Naoumova RP. Mechanisms of disease: genetic causes of familial hypercholesterolemia. Nat Clin Pract Cardiovasc Med. 2007;4(4):214-25.

27. Myant NB. Familial defective apolipoprotein B-100: a review, including some comparisons with familial hypercholesterolaemia. Atherosclerosis. 1993:104(1):1-18.

28. Abifadel $M$, Varret $M$, et al. Mutations in PCSK9 cause autosomal dominant hypercholesterolemia. Nat Genet. 2003;34(2):154-6.

29. Fahed AC, Nemer GM. Familial hypercholesterolemia: the lipids or the genes. Nutr Metab (Lond). 2011;8(1):23.

30. Lambert G, Charlton F, Rye KA, Piper DE. Molecular basis of PCSK9 function. Atherosclerosis. 2009;203(1):1-7.

31. Blom DJ, Hala T, et al. A 52-week placebo-controlled trial of evolocumab in hyperlipidemia. New Engl J Med. 2014;370(19):1809-19.

32. Soutar AK, Naoumova RP, Traub LM. Genetics, clinical phenotype, and molecular cell biology of autosomal recessive hypercholesterolemia. Arterioscler Thromb Vasc Biol. 2003;23(11):1963-70.

33. Garcia CK, Wilund K, et al. Autosomal recessive hypercholesterolemia caused by mutations in a putative LDL receptor adaptor protein. Science. 2001;292(5520):1394-8

34. Defesche JC, Lansberg PJ, et al. Advanced method for the identification of patients with inherited hypercholesterolemia. Semin Vasc Med. 2004;4(1):59-65.

35. Simon Broome Register Group. Risk of fatal coronary heart disease in familial hypercholesterolaemia. BMJ. 1991;303(6807):893-6.

36. Hsia SH, Connelly PW, Hegele RA. Genetic diagnosis of familial hypercholesterolemia in affected relatives using pedigree tracing. Clin Biochem. 1996;29(4):371-7.

37. Graham CA, Mcllhatton BP, et al. Genetic screening protocol for familial hypercholesterolemia which includes splicing defects gives an improved mutation detection rate. Atherosclerosis. 2005:182(2):331-40.

38. Al-Rasadi K, Al-Waili K, et al. Criteria for Diagnosis of Familial Hypercholesterolemia: A Comprehensive Analysis of the Different Guidelines, Appraising their Suitability in the Omani Arab Population. Oman Med J. 2014:29(2):85-91.

39. Damgaard D, Larsen ML, et al. The relationship of molecular genetic to clinical diagnosis of familial hypercholesterolemia in a Danish population. Atherosclerosis. 2005;180(1):155-60. 
40. Weng SF, Kai J, et al. Improving identification of familia hypercholesterolaemia in primary care: Derivation and validation of the familial hypercholesterolaemia case ascertainment tool (FAMCAT). Atherosclerosis. 2015:238(2):336-43.

41. Talmud P, Shah S, et al. Use of low-density lipoprotein cholesterol gene score to distinguish patients with polygenic and monogenic familial hypercholesterolaemia: a case-control study. Lancet. 2013;381 (9874):1293-301.

42. Veerkamp $M$, deGraaf J, et al. Diagnosis of familial combined hyperlipidaemia based on lipid phenotype expression in 32 families Results of a 5-Year Follow-Up Study. Arterioscler Thromb Vasc Biol. 2002;22(2):274-82

43. Nordestgaard B, Chapman MJ, et al. Lipoprotein(a) as a cardiovascular risk factor: current status. Eur Heart J. 2010;31(23):2844-53

44. National Institute for Health and Clinical Excellence (NICE). Familial Hypercholesterolaemia - Costing Report: Implementing NICE guidance. 2009. pp.1-42. https://www.nice.org.uk/guidance/cg71/resources/familialhypercholesterolaemia-costing-report2. Accessed 12 Oct 2015.

45. Versmissen J, Oosterveer DM, et al. Efficacy of statins in familial hypercholesterolaemia: a long term cohort study. BMJ. 2008;337:a2423.

46. Preiss D, Seshasai SRK, et al. Risk of incident diabetes with intensive-dose compared with moderate-dose statin therapy: a meta-analysis. JAMA. 2011; 305(24):2556-64

47. Pijlman $\mathrm{AH}$, Huijgen $\mathrm{R}$, et al. Evaluation of cholesterol lowering treatment of patients with familial hypercholesterolemia: a large cross-sectional study in The Netherlands. Atherosclerosis. 2010;209(1):189-94.

48. Reiner Z. Resistance and intolerance to statins. Nutr Metab Cardiovasc Dis. 2014;24(10):1057-66.

49. Galema-Boers JMH, Lenzen MJ, et al. Predicting non-adherence in patients with familial hypercholesterolemia. Eur J Clin Pharmacol. 2014;70(4):391-7.

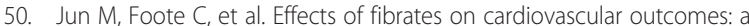
systematic review and meta-analysis. Lancet. 2010;375(9729):1875-84.

51. Ganji S, Kamanna V, Kashyap M. Niacin and cholesterol: role in cardiovascular disease (review). J Nutr Biochem. 2003;14(6):298-305.

52. Shek A, Ferrill M. Statin-fibrate combination therapy. Ann Pharmacother. 2001;35(7-8):908-17.

53. Guyton J, Capuzzi D. Treatment of hyperlipidemia with combined niacin-statin regimens. Am J Cardiol. 1998:82(12):82U-4.

54. Austin MA, Zimmern RL, Humphries SE. High "population attributable fraction" for coronary heart disease mortality among relatives in monogenic familial hypercholesterolemia. Genet Med. 2002;4(4):275-8.

55. World Health Organization. The International Classification of Diseases (ICD), 1990. http://apps.who.int/classifications/icd10/browse/2016/en\#/E78.0. Accessed 26 Sept 2015.

56. Marks D, Wonderling D, et al. Cost effectiveness analysis of different approaches of screening for familial hypercholesterolaemia. BMJ. 2002 324(7349):1303.

57. Bhatnagar D, Morgan J, et al. Outcome of case finding among relatives of patients with known heterozygous familial hypercholesterolaemia. Br Med J. 2000:321(7275):1497.

58. Starr B, Hadfield SG, et al. Development of sensitive and specific age-and gender-specific low-density lipoprotein cholesterol cutoffs for diagnosis of first-degree relatives with familial hypercholesterolaemia in cascade testing. Clin Chem Lab Med. 2008;46:791-803.

59. Umans-Eckenhausen MA, Defesche JC, et al. Review of first 5 years of screening for familial hypercholesterolaemia in the Netherlands. Lancet. 2001;357(9251):165-8.

60. Bell DA, Pang J, et al. Effectiveness of genetic cascade screening for familial hypercholesterolaemia using a centrally co-ordinated clinical service: An Australian experience. Atherosclerosis. 2015;239(1):93-100.

61. Jannes CE, Santos RD, et al. Familial hypercholesterolemia in Brazil: Cascade screening program, clinical and genetic aspects. Atherosclerosis. 2015;238(1):101-7.

62. Hadfield SG, Horara S, et al. Family tracing to identify patients with familial hypercholesterolaemia: the second audit of the Department of Health Familial Hypercholesterolaemia Cascade Testing Project. Ann Clin Biochem. 2009;46(1):24-32.

63. Marks D, Thorogood M, et al. Cascade screening for familial hypercholesterolaemia: implications of a pilot study for national screening programmes. J Med Screen. 2006;13(3):156-9.

64. Ned RM and Sijbrands EJ. Cascade screening for familial hypercholesterolemia (FH). PLoS Curr. 2011;3:RNN1238.
65. Morris JK, Wald DS, Wald NJ. The evaluation of cascade testing for familial hypercholesterolemia. Am J Med Genet A. 2012;158(1):78-84.

66. Green NS, Dolan SM, Murray TH. Newborn Screening: Complexities in Universal Genetic Testing. Am J Public Health. 2006;96(11):1955-9.

67. Anttila A, Ronco G, et al. Cervical cancer screening programmes and policies in 18 European countries. Brit J Cancer. 2004;9(5):935-41.

68. Farrell PM, Kosorok MR, et al. Early diagnosis of cystic fibrosis through neonatal screening prevents severe malnutrition and improves long-term growth. Pediatrics. 2001;107(1):1-13.

69. Waisbren SE, Albers S, et al. Effect of expanded newborn screening for biochemical genetic disorders on child outcomes and parental stress. JAMA. 2003;290(19):2564-72.

70. Finley-Austin MJ, Kreiner T. Integrating genomics technologies in health care: practice and policy challenges and opportunities. Physiol Genomics. 2002:8(1):33-40.

71. Watts $G$, Gidding $S$, et al. Integrated guidance on the care of familial hypercholesterolaemia from the International FH Foundation. Int J Cardiol. 2014;171(3):309-25.

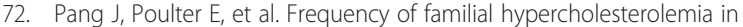
patients with early-onset coronary artery disease admitted to a coronary care unit. J Clin Lipidol. 2015:9(5):703-8.

73. DeMott K, Nherera L, et al. Clinical Guidelines and Evidence Review for Familial hypercholesterolaemia: the identification and management of adults and children with familial hypercholesterolaemia. London: National Collaborating Centre for Primary Care and Royal College of General Practitioners. ROYAL COLLEGE OF GENERAL PRACTITIONERS; 2008.

74. O'Brien EC, Roe MT, et al. Rationale and design of the familial hypercholesterolemia foundation Cascade Screening for Awareness and Detection of Familial Hypercholesterolemia registry. Am Heart J. 2014;167(3):342-9.

75. Gray J, Jaiyeola A, et al. Identifying patients with familial hypercholesterolaemia in primary care: an informatics-based approach in one primary care centre. Heart. 2008;94(6):754-8.

76. Wald DS, Bestwick JP, Wald NJ. Child-parent screening for familial hypercholesterolaemia: screening strategy based on a meta-analysis. BMJ. 2007;335(7620):599.

77. Faiz F, Allcock $R$, et al. Detection of variations and identifying genomic breakpoints for large deletions in the LDLR by lon Torrent semiconductor sequencing. Atherosclerosis. 2013;230(2):249-55.

78. Hinchcliffe $M$, Le $H$, et al. Diagnostic validation of a familial hypercholesterolaemia cohort provides a model for using targeted next generation DNA sequencing in the clinical setting. Pathol J RCPA. 2014;46(1):60-8.

79. Maglio C, Mancina B, et al. Genetic diagnosis of familial hypercholesterolaemia by targeted next-generation sequencing. J Intern Med. 2014;276(4):396-403.

80. Vandrovcova J, Thomas $\mathrm{E}$, et al. The use of next-generation sequencing in clinical diagnosis of familial hypercholesterolemia. Gen Med. 2013; 15(12):948-57.

81. Stein E, Raal F. Polygenic familial hypercholesterolaemia: does it matter? Lancet. 2013;381(9874):1255-7.

82. Raal $F$, Stein $E$. What matters most in pediatric familial hypercholesterolemia, genotype or phenotype? J Lipid Res. 2014;55(5):793-4.

83. Goldberg A, Hopkins P, et al. Familial hypercholesterolemia: screening, diagnosis and management of pediatric and adult patients: clinical guidance from the National Lipid Association Expert Panel on Familial Hypercholesterolemia. J Clin Lipidol. 2011;5(3):133-40.

84. Heart UK. Saving lives, saving families the health, social and economic advantages of detecting and treating familial hypercholesterolaemia (FH). 2012. www.heartuk.org.uk/files/uploads/documents/HUK_ SavingLivesSavingFamilies_FHreport_Feb2012.pdf. Accessed 15 Jul 2015

85. National Institute for Health and Clinical Excellence (NICE), 2013. Judging whether public health interventions offer value for money. https://www. nice.org.uk/advice/lgb10/chapter/judging-the-cost-effectiveness-of-publichealth-activities. Accessed 26 Sept 2015

86. Norsworthy $P$, et al. Targeted genetic testing for familial hypercholesterolaemia using next generation sequencing: a population-based study. BMC Med Genet. 2014;15(70):1.

87. Burn J. Should we sequence everyone's genome? Yes. BMJ. 2013;346:f3133.

88. Langslet G, Ose L. Screening methods in the diagnosis and assessment of children and adolescents with familial hypercholesterolemia. Expert Rev Cardiovasc Ther. 2013;11(8):1061-6. 
89. Krawczak M, Cooper DN, Schmidtke J. Estimating the efficacy and efficiency of cascade genetic screening. Am J Hum Genet. 2001;69(2):361-70.

90. Wetterstrand KA. DNA Sequencing Costs: Data from the NHGRI Genome Sequencing Program (GSP). https://www.genome.gov/sequencingcosts. Accessed 15 Jul 2015.

91. Hayden EC. The \$1,000 genome. Nature. 2014;507(7492):294-5.

92. Meulenkamp TM, Tibben $\mathrm{A}$, et al. Predictive genetic testing for cardiovascular diseases: impact on carrier children. Am J Med Genet A. 2008;146(24):3136-46.

93. Davidson MH. Dyslipidaemia: PCSK9 antibodies: a dividend of the genomics revolution. Nat Rev Cardiol. 2013;10(11):618-9.

94. Lagace TA. PCSK9 and LDLR degradation: regulatory mechanisms in circulation and in cells. Curr Opin Lipidol. 2014;25(5):387-93.

95. Williams RR, Hunt SC, et al. Diagnosing heterozygous familial hypercholesterolemia using new practical criteria validated by molecular genetics. Am J Cardiol. 1993;72(2):171-6.

Submit your next manuscript to BioMed Central and we will help you at every step:

- We accept pre-submission inquiries

- Our selector tool helps you to find the most relevant journal

- We provide round the clock customer support

- Convenient online submission

- Thorough peer review

- Inclusion in PubMed and all major indexing services

- Maximum visibility for your research

Submit your manuscript at www.biomedcentral.com/submit
Biomed Central 